Instituto Internacional de Investigación y Desarrollo Tecnológico Educativo INDTEC, C.A. DOI: https://doi.org/10.29394/Scientific.issn.2542-2987.2020.5.17.1.19-38

OAI-PMH: http://www.indteca.com/ojs/index.php/Revista Scientific/oai

Artículo Original / Original Article

\title{
Programa de capacitación para desarrollar competencias investigativas, dirigido a los docentes en su accionar pedagógico
}

\author{
Autor: Alirio Alejandro Tua García \\ Unidad Educativa Nacional San Francisco Javier, UENSFJ \\ aliriotua@gmail.com \\ Lara, Venezuela \\ https://orcid.org/0000-0002-9240-0487
}

Resumen

En la sociedad actual, es inevitable la trascendencia de la investigación en todos los ámbitos del conocimiento, pero sin lugar a duda es en los procesos educativos donde radica su auge. Por ello, los programas de capacitación docente en competencias investigativas son imprescindibles para alcanzar una calidad educativa. Este artículo se fundamentó en los postulados de Freire (2004); Stenhouse (2007); Andrade (2015); Díaz (2017); y Gil (2017), entre otros. El objetivo se basó en diseñar un programa de capacitación para desarrollar competencias investigativas dirigido a los docentes en su accionar pedagógico de la Unidad Educativa Nacional San Francisco Javier en Barquisimeto, municipio Iribarren, estado Lara, Venezuela. Se utilizó una metodología de campo descriptiva con modalidad proyectiva. En cuanto a la población de estudio, esta estuvo conformada por 50 docentes. Para la técnica de recolección de datos, se manejó en un cuestionario estructurado con alternativas de respuestas, validado mediante la técnica de juicio de expertos, y calculada su confiablidad con el coeficiente Alpha de Cronbach, generando 0.96 de alta confiabilidad. Los resultados fueron analizados y tabulados, demostrando la necesidad de un programa de capacitación para desarrollar competencias investigativas. Como conclusión, se determinó que los planteamientos y los postulados permitieron justificar el diseño del programa de capacitación.

Palabras clave: programa de formación de docentes; investigación; calidad de la educación; metodología.

Cómo citar este artículo:
Tua, A. (2020). Programa de capacitación para desarrollar competencias investigativas, dirigido
a los docentes en su accionar pedagógico. Revista Scientific, 5(17), 19-38, e-ISSN: 2542-2987.
Recuperado de: https://doi.org/10.29394/Scientific.issn.2542-2987.2020.5.17.1.19-38

Fecha de Recepción: 02-03-2020
Fecha de Aceptación: 05-05-2020
Fecha de Publicación: 05-08-2020 
OAI-PMH: http://www.indteca.com/ojs/index.php/Revista_Scientific/oai

Artículo Original / Original Article

\title{
Training program to develop investigative skills, aimed at teachers in their pedagogical actions
}

\begin{abstract}
In today's society, the importance of research in all areas of knowledge is inevitable, but it is undoubtedly in educational processes that its rise lies. Therefore, teacher training programs in investigative skills are essential to achieve educational quality. This article was based on the postulates of Freire (2004); Stenhouse (2007); Andrade (2015); Díaz (2017); and Gil (2017), among others. The objective was based on designing a training program to develop investigative skills aimed at teachers in their pedagogical actions of the San Francisco Javier National Educational Unit in Barquisimeto, Iribarren municipality, Lara state, Venezuela. A descriptive field methodology with a projective modality was used. As for the study population, it was made up of 50 teachers. For the data collection technique, it was managed in a structured questionnaire with alternative answers, validated using the expert judgment technique, and its reliability was calculated with the Cronbach's Alpha coefficient, generating 0.96 of high reliability. The results were analyzed and tabulated, demonstrating the need for a training program to develop investigative skills. In conclusion, it was determined that the approaches and the postulates allowed to justify the design of the training program.
\end{abstract}

Keywords: teacher education curriculum; investigation; educational quality; methodology.

\footnotetext{
How to cite this article:

Tua, A. (2020). Training program to develop investigative skills, aimed at teachers in their pedagogical actions. Revista Scientific, 5(17), 19-38, e-ISSN: 2542-2987. Recovered from: https://doi.org/10.29394/Scientific.issn.2542-2987.2020.5.17.1.19-38
}

Date Received: 02-03-2020
Date Acceptance:

05-05-2020
Date Publication:

05-08-2020 


\section{Introducción}

La investigación educativa promueve al docente el anhelo de participar consciente en diversos planes educativos con la finalidad de ampliar su capacitación, no sólo a nivel de conocimientos relacionados con su ámbito de acción, sino, también como una herramienta básica para alcanzar el éxito de su participación en el campo educativo y profesional. A medida que se dé el proceso de actualización, se promoverá el desarrollo de las habilidades mentales y emocionales, a la vez que se forman conocimientos, destrezas y valores permitiéndole al docente reconocer sus potencialidades en el campo investigativo.

Por consiguiente, es necesario que la capacitación del docente se proyecte hacia varios escenarios y no se enmarque sólo a nivel institucional, de manera que éste pueda aplicar, desde su sitio de trabajo una serie de prácticas basadas en la investigación. En consecuencia, el presente estudio, incluye el diseño de un programa para desarrollar competencias investigativas, de manera que el maestro demuestre habilidades en cuanto a: conocimientos, destrezas y rasgos personales propios de un investigador. En particular, Tabón (2009), citado por Andrade (2015), señala que:

Son procesos complejos de desempeño con idoneidad en determinados contextos, integrando diferentes saberes (saber ser, saber hacer, saber conocer y saber convivir), para realizar actividades y/o resolver problemas con sentido de reto, motivación, flexibilidad, creatividad, comprensión y emprendimiento, dentro de una perspectiva de procesamiento meta cognitivo, mejoramiento continuo y compromiso ético, con la meta de contribuir al desarrollo personal, la construcción y afianzamiento del tejido social, la búsqueda continua del desarrollo económico-empresarial sostenible, y el cuidado y protección del ambiente y de las especies vivas (pág. 21).

Es así, como las competencias investigativas forman los componentes propios en la formación docente. Destaca Freire (2004): que "no hay 
enseñanza sin investigación ni investigación sin enseñanza" (pág. 17). Asimismo, el docente encargado de orientar a sus estudiantes en el proceso educativo, debe mostrar un perfil especial, en la medida en que la investigación sea su objetivo profesional y personal, ya que éste no es sólo la autoridad que domina el conocimiento y que lo trasmite, hoy en día se perfeccionan nuevos roles, entre los cuales destacan, partiendo del punto de vista de Stenhouse (2007): "la innovación educativa, la elevación de la calidad de la enseñanza, la permanente actualización del profesorado y la autonomía de funcionamiento de los centros escolares hacen que no sólo sea aplicable, sino necesario..." (pág. 18).

De acuerdo con lo anterior, se interpreta que la participación del docente investigador en el proceso educativo promueve el desarrollo de las habilidades investigativa del estudiantado, a la vez que forma conocimientos, destrezas y valores permitiendo al educando reconocer sus potencialidades preparándolos para que en un futuro cercano solucionen problemas personales y grupales, debe ofrecer un proceso investigativo con una visión interactiva que permita vivenciar el aprendizaje y llevar a la práctica las destrezas adquiridas.

Hay que destacar, en materia educativa, la investigación está relacionada con todas las disciplinas y ciencias del saber. Al respecto, Organización de las Naciones Unidas para la Educación, la Ciencia y la Cultura (UNESCO, 1998), plantea:

Promover, generar y difundir conocimientos por medio de la investigación y, como parte de los servicios que ha de prestar a la comunidad, proporcionar las competencias técnicas adecuadas para contribuir al desarrollo cultural, social y económico de las sociedades, fomentando y desarrollando la investigación científica y tecnológica a la par que la investigación en el campo de las ciencias sociales, las humanidades y las artes creativas (pág. 4).

Es evidente, en el día a día se construyen y se reconstruyen 
conocimientos, que de una u otra forma están relacionados con la vida misma, y que por medio de la comunicación se enriquece la experiencia pedagógica de ese entorno específico, el docente al investigar conseguirá múltiples casos y diferentes formas de abordarlo, de allí que se diga que el investigador debe ser creativo, ya que por más parecidos o coincidencias que emerjan en sus estudios cada uno tiene características peculiares.

No obstante, Delgado y Alfonzo (2019), afirman que:

El papel docente-investigador implica reflexionar permanentemente, asumir una actitud crítica sobre lo aprendido en su formación y notificar saberes con sus pares y estudiantes, para establecer sinergias entre lo que se aprende e investiga, además sobre lo que se enseña y el para qué. Imparcialmente, la figura del docente-investigador lleva a considerar al sujeto como un representante reflexivo capaz de articular la praxis docente con la investigación (págs. 203-204).

A tal efecto, el docente en la actualidad debe tener una actitud investigativa frente a los obstáculos que se le pueden presentar en su praxis pedagógica, ya que su objetivo es mejorar su labor cuando enfrente el reto de profundizar sobre problemas que surgen en su rutina diaria de clase, para que luego aplique estrategias y pueda solventarlos.

Frente a esto, Hernández (2009): menciona el docente investigador, es un "sujeto de la educación, con un ethos orientado hacia su función de educador, formador e investigador [...]" (pág. 14); generalmente el docente ha sido utilizado en los centros educativos para qué aplique propuestas educativas, pensadas por otros pedagogos, no son necesidades sentidas por él en su área de trabajo, ni ha participado en la investigación y elaboración de las propuestas.

En este mismo contexto, la Organización de las Naciones Unidas para la Educación, la Ciencia y la Cultura (UNESCO, 2015), explica que: "una reciente iniciativa gubernamental ha revolucionado la investigación 
OAI-PMH: http://www.indteca.com/ojs/index.php/Revista_Scientific/oai

\section{Artículo Original / Original Article}

universitaria estableciendo un Organismo Federal para las Organizaciones de Investigación encargado de financiar y gestionar la propiedad de los institutos de investigación" (pág. 30). Frecuentemente, se ve al docente como un aplicador de recetas, minimizándolo en su rol, cuando en realidad está llamado a cumplir funciones de gran relevancia, una de ellas es el de ser investigador, puesto que su horizonte está basado en las necesidades, intereses e iniciativa de los educandos.

Por tal motivo, es prioritario preparar al pedagogo en el campo de la investigación educativa, puesto que, es él quien conoce la realidad en la que está inmerso su grupo de alumnos y es quien puede dar posibles respuestas o soluciones a la problemática que detecta en el aula para satisfacer inquietudes socioeducativas del estudiantado que atiende.

Evidentemente, la acción del docente en su aula de clase es un factor que afecta o mejora la calidad educativa, puesto que debe prepararse constantemente para estar a la altura de los diferentes roles que le corresponden, bien sea como modelo, mediador, transmisor, técnico, innovador, precisar cambios, resolver problemas y mejorar la realidad educativa, comunitaria y científica e inclusive en su rol de evaluador de los procesos de aprendizajes de sus alumnos; para lo cual debe valerse de diversas técnicas e instrumentos.

Sobre este particular, Maldonado (2015), citado por Díaz (2017), sostiene que:

La escasa importancia que los docentes le están dando a su capacitación está repercutiendo de manera directa en la formación de los niños y jóvenes del país, es lamentable observar cómo los entornos educativos en Venezuela son desalentadores, monótonos, apáticos, parece que a nadie le importa constituir una comunidad del conocimiento (pág. 8).

La educación requiere de docentes capacitados e interesados en las necesidades sociales y el mejoramiento de la calidad educativa. Todos los 
avances, innovaciones y reformas en general llegan a las instituciones educativas gracias al perfeccionamiento docente, bien sea por iniciativa propia o por políticas educativas de Estado.

\section{El Problema}

Por otra parte, las competencias investigativas del docente son herramientas básicas para alcanzar éxito en su labor y su accionar pedagógico. Cabe resaltar que, Saby (2012), expresa que: “[...] el rol del docente depende del modo como se asuma la docencia investigativa y la relación entre investigación y enseñanza [...]" (pág. 26).

En este sentido, se tiene el caso concreto de la Unidad Educativa Nacional "San Francisco Javier" en Barquisimeto, municipio Iribarren, estado Lara, Venezuela, los directivos manifiestan su preocupación por la falta de habilidades investigativa en los docentes, causando baja producciones intelectuales, en su accionar pedagógico tradicional en el campo educativo.

De igual manera, en entrevistas informales con los docentes manifiestan verbalmente que las investigaciones no les llama la atención, prefieren dar clase tradicionales, obviando así las competencias y herramientas investigativas, lo cuales son necesarias para en el campo educativo.

En conversaciones informales agregan los profesionales de la enseñanza: la investigación es una imposición que me agobia, no me siento preparada para investigar, yo investigue en mi época, cuando era estudiante, la investigación es muy difícil, eso es para los docentes universitarios, investigar exige una serie de conocimientos que quizás en estos momentos no poseo, a veces realizó una que otra investigación, pero no tan rigurosa, pues me faltan herramientas investigativas.

La situación descrita en los párrafos precedentes es considerada problemática por el investigador, pues es necesario las competencias 
investigativas en el accionar pedagógico de los docentes que contribuya en el campo educativo y pueda proyectar este saber a los estudiantes en el quehacer investigativo.

\subsection{Objetivo de la Investigación}

Diseñar un programa de capacitación para desarrollar competencias investigativas dirigido a los docentes en su accionar pedagógico en la Unidad Educativa Nacional San Francisco Javier de Barquisimeto, municipio Iribarren, estado Lara, Venezuela.

\section{Contexto temático y abordaje teórico}

\subsection{Antecedentes de la investigación}

Los estudios previos, son investigaciones realizadas que permiten transformaciones sociales y que facilitan el abordaje de problemáticas. Los antecedentes, conforme a la Universidad Pedagógica Experimental Libertador (UPEL, 2016): "comprende una revisión de los trabajos previos realizados sobre el problema en estudio y (o) de la realidad contextual [...]" (pág. 34); a continuación, se presenta algunas:

En esa misma línea, Mera (2017a): realizó un trabajo, que tuvo como objetivo fortalecer el Aprendizaje de Investigación de los docentes a través de un Programa Educativo Investigativo. Se empleó una metodología de tipo descriptivo propositivo, de diseño no experimental y transversal. La población la conformaron veinte (20) docentes. La técnica utilizada fue la encuesta y como instrumento un cuestionario.

Paralelamente, Mera (2017b): concluyó que los docentes participantes tienen un aprendizaje de investigación casi nulo, debido a la ausencia de una cultura de investigación científica, por lo cual se diseñó una Propuesta de un Programa Educativo Investigativo conformado por un conjunto de talleres.

Por su parte, Araujo (2016), quien realizó una investigación descriptiva 
con diseño de campo, en el que tuvo como propósito: "analizar la formación de los docentes de educación media general desde el enfoque por competencias de los profesores" (pág. 186). La población de estudio consintió en cincuenta (50) docentes encuestados, en los que se determinó que tienen una enseñanza tradicional donde lo primordial son los contenidos de forma conductual. Además, los encuestados carecen de teorías actualizadas y competencias inherentes en su práctica y su accionar docente.

Cabe agregar que, estos estudios son significativos para este trabajo, ya que presenta énfasis en el enfoque por competencias y la investigación, convirtiéndose en un verdadero reto para el docente, incluir estos dos elementos en su accionar pedagógico para obtener una calidad en el campo educativo con miras a crear nuevas situaciones de enseñanza.

\subsection{Competencias Investigativas}

La educación busca formar personas competente y críticas que tengan una buena capacidad de adaptarse a los cambios y que puedan interpretar la información disponible y para ellos los docentes en servicio deben estar en constante actualizaciones.

Refiere Jaik (2013), que las competencias investigativas son: "[...] el conjunto de conocimientos, actitudes, habilidades y destrezas necesarias para llevar a cabo la elaboración de un trabajo de investigación [...]” (pág. 24). De acuerdo con lo expresado, las competencias investigativas implican conocimientos, valores, y responsabilidades para llevar a cabo un estudio con rigor científico.

En esta misma perspectiva, Gallardo (2003), las define como:

[...] Una configuración construida y desarrollada por los investigadores en su contexto y ejercicio profesional. Incluye la solución de problemas del entorno donde muestra suficientes habilidades en el arte de investigar, a través de la cual logra incorporar lo nuevo que permitirá transformar la realidad, para 
OAI-PMH: http://www.indteca.com/ojs/index.php/Revista_Scientific/oai

Artículo Original / Original Article

ello utiliza la tecnología; además comunica y dirige el proceso hacia la obtención de resultados de significativo impacto económico, científico, social y/o medio ambiental (pág. 11).

Esto indica que, es necesario que el docente posea estrategias para afianzar las competencias investigativas y orientar al estudiante en sus conocimientos. El docente de hoy debe estar dispuesto a reaprender cada día, buscar las mejores estrategias que le permitan organizar sus acciones educativas, que le brinde la posibilidad de cumplir con su rol de agente de cambio.

\subsection{Programa de Capacitación}

Es ineludible enfrentar cambios y retos permanentes por lo que se hace indispensable una transformación de los procesos pedagógicos aplicados a la práctica pedagógica mediante la aplicación de un programa. En este sentido, el concepto de programa lo afirma Gil (2017):

[...] Como el anticipo de lo que se planea realizar en algún ámbito o circunstancia; el temario que se ofrece para un discurso; la presentación y organización de las materias de un cierto curso o asignatura; y la descripción de las características o etapas en que se organizan determinados actos o espectáculos artísticos (pág. 229).

Los programas son una necesidad para la capacitación de todo individuo, en especial para la formación docente en su propio accionar pedagógico que busca la calidad educativa, por ello, los programas de capacitación representan alternativas que responden a la necesidad de cada uno de los actores del proceso educativo.

Al mismo tiempo, Arechavala (2011), asevera que:

[...] En Latinoamérica siguen, en su mayoría, sin desarrollar significativamente la investigación, y desconociendo la repercusión que esto tiene en la calidad de la docencia y en la capacidad de la universidad para contribuir de manera directa 
al bienestar económico y social de la región en la que opera (pág. 45).

De este modo, el autor alude a que existen pocas instituciones y personal con logros investigativos. Desde esta mirada los centros educativos están forzadas a revisar su proceso investigativo para replantearse las metas al hacer investigación, coadyuvando a la sociedad a mejorar su calidad de vida, por medio de actividades creativas e innovadoras que generen conocimiento en la búsqueda de la comprensión de una realidad compleja.

\subsection{Capacitación Docente}

En termino de Grados (2010), citado por Valencia (2017), "un plan de capacitación es un método complejo e integral que se hace necesario y obligatorio dentro de una organización" (pág. 160). Se requiere dotar al docente de herramientas metodológicas-didácticas para garantizar que los estudiantes aprendan más y mejor, pero es necesario que la capacitación docente lleve un seguimiento real, es decir, en su relación directa con el mejoramiento de la propuesta de enseñanza. El docente es un medio clave para mejorar la calidad educativa, pero indiscutiblemente debe primero mejorar los conocimientos y destrezas propios para que pueda transferir los aprendizajes de manera significativa.

Cabe resaltar que, la capacitación en relación a las competencias investigativas, facilitará al educador desarrollar el juicio crítico de la realidad educativa para manejar con eficiencia las incertidumbres y contradicciones que se producen durante el proceso educativo; emplear herramientas, tales como la indagación de la comunidad, el diálogo reflexivo, formulación y reformulación del problema, concebir a los actores desde una perspectiva holística como un todo dinámico que obedece a una lógica propicia de organización, funcionalidad, significancias y entrenarse en registro de 
OAI-PMH: http://www.indteca.com/ojs/index.php/Revista_Scientific/oai

\section{Artículo Original / Original Article}

información, codificación e interpretación en categorías y teorías que la sustentan.

\section{Metodología}

El presente estudio es una investigación cuantitativa, de campo, no experimental y descriptiva, con modalidad proyectiva, elaborando un programa de capacitación para desarrollar competencias investigativas dirigido a los docentes en su accionar pedagógico en la Unidad Educativa Nacional "San Francisco Javier" de Barquisimeto, municipio Iribarren, estado Lara, Venezuela.

Se empleó la técnica de encuesta, y el instrumento un cuestionario impreso con escala tipo Likert: Muy Necesario (MN), Necesario (N) Poco Necesario (PN), No Necesario (NN). Acorde con esto, Arias (2012), complementa que es: "una técnica que pretende obtener información que suministra un grupo o muestra de sujetos acerca de sí mismos, o en relación con un tema en particular" (pág. 72); dado en este caso el diseño de un programa de capacitación para desarrollar competencias investigativas.

La validación se ejecutó a través de juicio de expertos y probada su confiabilidad mediante el estadístico Alfa de Cronbach, obteniendo 0,96 (alta confiabilidad). Posteriormente, se aplicó a diez sujetos de estudio, quienes son docentes en el Liceo Bolivariano "Ezequiel Bujanda" en Barquisimeto, estado Lara, Venezuela, los cuales dieron su consentimiento para la obtención de los datos.

El cumplimiento de los objetivos propuestos en este estudio, se siguieron los siguientes pasos:

1. Detección del problema.

2. Revisión bibliográfica.

3. Redacción del planteamiento de problema.

4. Elaboración de antecedentes, bases teóricas y fundamentación legal. 
5. Estructura del marco metodológico.

6. Diseño del instrumento de recolectar los datos.

7. Validación del instrumento.

8. Consentimiento Informado para la obtención de los datos.

9. Aplicación de pruebas piloto.

10. Verificación de la Confiabilidad del instrumento.

11. Organización de los resultados.

12. Análisis y discusión de los resultados.

13. Formulación de las conclusiones y recomendaciones.

\section{Resultados}

Se exponen los resultados del estudio analítico de investigación a través de la información recogida en el instrumento de recolección de datos dirigido a los cincuenta (50) docentes de la Unidad Educativa Nacional "San Francisco Javier" en Barquisimeto, municipio Iribarren, estado Lara, Venezuela.

En la tabla 1, se sintetiza la información recolectada por los docentes de la institución antes mencionada, con el fin recabar datos que permitan conocer la necesidad educativa presentada.

Tabla 1. Valores en frecuencias y porcentajes de la respuesta de los sujetos encuestados con el indicador Destreza y la dimensión Necesidad.

\begin{tabular}{|c|c|c|c|c|c|c|c|c|c|}
\hline \multirow{2}{*}{\multicolumn{2}{|c|}{ Proposición }} & \multicolumn{2}{|c|}{ MN } & \multicolumn{2}{|c|}{$\mathrm{N}$} & \multicolumn{2}{|c|}{ PN } & \multicolumn{2}{|c|}{ NN } \\
\hline & & ᄃ & 0 & 5 & 0 & 5 & or & ᄃ & \\
\hline 1 & $\begin{array}{l}\text { Es impredecible un programa de capacitación que } \\
\text { promueva competencias investigativas en el accionar } \\
\text { pedagógico de los docentes }\end{array}$ & 50 & 100 & 0 & 0 & 0 & 0 & 0 & 0 \\
\hline 2 & $\begin{array}{l}\text { El programa de capacitación facilitaría herramientas } \\
\text { para la redacción de informes investigativos. }\end{array}$ & 45 & 90 & 5 & 10 & 0 & 0 & 0 & 0 \\
\hline 3 & $\begin{array}{l}\text { El programa de capacitación permitiría desarrollar } \\
\text { competencia investigativa en su accionar diario }\end{array}$ & 48 & 96 & 2 & 4 & 0 & 0 & 0 & 0 \\
\hline 4 & $\begin{array}{l}\text { El programa de capacitación facilitaría herramientas } \\
\text { para evaluar el proceso educativo de sus estudiantes } \\
\text { al aplicar diversas técnicas e instrumentos de } \\
\text { investigación }\end{array}$ & 47 & 94 & 3 & 6 & 0 & 0 & 0 & 0 \\
\hline
\end{tabular}


OAI-PMH: http://www.indteca.com/ojs/index.php/Revista_Scientific/oai

Artículo Original / Original Article

\begin{tabular}{|c|l|l|l|l|l|l|l|l|l|}
\hline 5 & $\begin{array}{l}\text { El programa de capacitación sobre competencias } \\
\text { ofrecerá habilidad metodológica. }\end{array}$ & 50 & 100 & 0 & 0 & 0 & 0 & 0 & 0 \\
\hline Valores Promedios & 48 & 96 & 2 & 4 & 0 & 0 & 0 & 0 \\
\hline
\end{tabular}

Fuente: El Autor (2020).

Se procede a detallar la data obtenida en la dimensión destrezas, se aprecia lo siguiente en la tabla 1, el ítem 1,100\%, de los docentes encuestados consideran como muy necesario un programa de capacitación que promueva competencias investigativas en el accionar pedagógico. En el ítem 2, se obtuvo que el $90 \%$ de los docentes consideran muy necesario un programa de capacitación que faciliten herramientas para la redacción de informes investigativos y el $10 \%$ piensa que es necesario dicho programa.

En cuanto al ítem 3, el 96\% de los docentes consideran que el programa de capacitación permitiría desarrollar competencia investigativa en su accionar diario y el $4 \%$ manifestaron que es necesario desarrollar competencia investigativa con un programa de capacitación. En el ítem 4, 94\% de los docentes creen que es muy necesario que el programa de capacitación facilite herramientas para evaluar el proceso educativo de sus estudiantes al aplicar diversas técnicas e instrumentos de investigación y el $6 \%$ considera que es necesario.

En el ítem 5, el $100 \%$ de los docentes creen que un programa de capacitación sobre competencias ofrecerá habilidad metodológica. En correspondencia, Machado (2008), citado por Aguirre y Benavides (2015), precisa la habilidad investigativa como: "el dominio de la acción que se despliega para solucionar tareas investigativas en el ámbito docente, laboral y propiamente investigativo" (pág. 7).

De allí los valores promedios obtenidos son los siguientes: $97 \%$ de los docentes considera muy necesario el programa de capacitación para desarrollar competencias investigativas y el $3 \%$ piensa que es necesario el 
programa de capacitación sobre competencias investigativas en su accionar pedagógico. Adicionalmente, González, Zerpa, Gutiérrez y Pirela (2007), mencionan que: "[...] cualquier profesional de la docencia vinculado al mundo de las instituciones, está llamado a desempeñar un papel clave como investigador de su propia práctica con la finalidad de mejorar su formación, su desempeño en el aula, escuela y comunidad [...]" (pág. 280).

\section{Conclusiones}

En cuanto al diagnóstico de la necesidad de un programa de capacitación para desarrollar las competencias investigativas dirigido a los docentes en su accionar pedagógico se determinó, la mayoría nunca emplea herramientas investigativas. Los planteamientos del análisis permitieron deducir y justificar el diseño del programa de capacitación en competencias investigativas dirigido a los docentes de la Unidad Educativa Nacional San Francisco Javier de Barquisimeto, municipio Iribarren, estado Lara, Venezuela, en función de optimizar la calidad investigativa de los participantes.

Asimismo, los resultados revelaron que los docentes encuestados son altamente receptivos y poseen expectativas favorables para realizar el programa de capacitación en competencias investigativas. De esta forma, se logró que el docente se actualice, aplique metodologías propias de la investigación científica, planifiquen y ejecuten técnicas e instrumentos que les sean útiles, tanto a nivel profesional como personal.

La validación del programa permitió hacer todos los ajustes que garantizaron el funcionamiento eficiente desde la óptica pedagógica y los potenciales usuarios. La validación de juicio de expertos y la validación de campo confirmaron categóricamente que el programa de capacitación para desarrollar competencias investigativas puede ser implementada por todos los docentes de cualquier casa de estudio.

De igual forma, con los resultados obtenidos en la presente 
investigación, se recomienda a la dirección del plantel realizar planificaciones e implementar seminarios, talleres o jornadas de capacitación docente en las diferentes áreas del saber empleando la investigación científica como herramienta para la indagación, detección de problemas y la búsqueda de soluciones en el accionar pedagógico del docente.

Se sugiere, al personal de la Unidad Educativa Nacional San Francisco Javier de Barquisimeto, municipio Iribarren, estado Lara, Venezuela, ejecutar al inicio de cada año escolar el programa propuesto sobre capacitación en competencias investigativas, para afianzar las habilidades de los participantes, de manera que se apliquen los conocimientos y destrezas investigativas.

\section{Referencias}

Aguirre, M., \& Benavides, S. (2015). Diagnóstico de las habilidades investigativas en los estudiantes de la carrera de pedagogía de la Universidad Politécnica Salesiana. Trabajo de titulación. Quito, Ecuador: Universidad Politécnica Salesiana. Recuperado de: https://dspace.ups.edu.ec/bitstream/123456789/9748/1/QT07538.pdf Andrade, E. (2015). Competencias investigativas del docente en educación matemática. Tesis de maestría. Carabobo, Venezuela: Facultad de Ciencias de la Educación de la Universidad de Carabobo. Recuperado de:

http://riuc.bc.uc.edu.ve/bitstream/123456789/1377/1/Eandrade.pdf Araujo, M. (2016). Formación del Docente de Educación Media General desde el Enfoque por Competencias. Revista Scientific, 1(2), 181199, e-ISSN: 2542-2987. Recuperado de:

https://doi.org/10.29394/scientific.issn.2542-2987.2016.1.2.11.181-199 Arechavala, R. (2011). Las universidades y el desarrollo de la investigación científica y tecnológica en México: una agenda de investigación. Revista de la educación superior, 40(158), 41-57, e- 
ISSN: 0185-2760. Recuperado de:

http://www.scielo.org.mx/scielo.php?script=sci arttext\&pid=S018527602011000200003\&lng=es

Arias, F. (2012). El proyecto de investigación: Introducción a la metodología científica. 6ta edición, ISBN: 980-07-8529-9. Caracas, Venezuela: Editorial Episteme, C.A.

Delgado, Y., \& Alfonzo, R. (2019). Competencias Investigativas del Docente Construidas durante la Formación Universitaria. Revista Scientific, 4(13), 200-220, e-ISSN: 2542-2987. Recuperado de:

https://doi.org/10.29394/Scientific.issn.2542-2987.2019.4.13.10.200$\underline{220}$

Díaz, C. (2017). La formación docente: un acercamiento al proceso de capacitación en la educación primaria. Mucuties Universitaria, 4(6), 4-11, ppi201402ME4558. Recuperado de:

http://erevistas.saber.ula.ve/index.php/mucuties/article/view/8461

Freire, P. (2004). Pedagogía de la Autonomía: Saberes necesarios para la práctica educativa. ISBN: 85-219-0243-3. Sao Paulo, Brasil: Paz e Terra, S.A.

Gallardo, O. (2003). Modelo de formación por competencia para investigadores. Revista Contexto \& Educação, 18(70), 9-25, e-ISSN: 2179-1309. Recuperado de:

https://www.revistas.unijui.edu.br/index.php/contextoeducacao/article/v iew/1141

Gil, M. (2017). Programa para padres en el Uso de Internet de sus Hijos. Revista Scientific, 2(6), 217-237, e-ISSN: 2542-2987. Recuperado de: https://doi.org/10.29394/scientific.issn.2542-2987.2017.2.6.11.217-237

González, N., Zerpa, M., Gutiérrez, D., \& Pirela, C. (2007). La investigación educativa en el hacer docente. Laurus, 13(23), 279-309, e-ISSN: 1315-883X. Recuperado de: 
OAI-PMH: http://www.indteca.com/ojs/index.php/Revista_Scientific/oai

Artículo Original / Original Article

https://www.redalyc.org/articulo.oa?id=76102315

Hernández, I. (2009). El docente investigador en la formación de profesionales. Revista Virtual Universidad Católica del Norte, (27), 121, e-ISSN: 0124-5821. Recuperado de:

https://www.redalyc.org/articulo.oa?id=194215432011

Jaik, A. (2013). Competencias Investigativas: Una mirada a la Educación Superior. Primera edición, ISBN: 978-607-9063-17-7. México: Editor Red Durango de Investigadores Educativos, A.C. - ReDIE.

Mera, H. (2017a,b). Propuesta de un Programa Educativo Investigativo para mejorar el aprendizaje de investigación de los docentes de la Institución Educativa Nº 00925 - "Santa Isabel" - Nivel Secundaria, del distrito de Nueva Cajamarca, provincia de Rioja, 2016. Tesis. Tarapoto, Perú: Universidad César Vallejo. Recuperado de: http://repositorio.ucv.edu.pe/bitstream/handle/UCV/12970/mera nh.pdf

Saby, J. (2012). El rol del docente-investigador en el marco de la investigación formativa. Revista PAPELES, 4(8), 23-30, ISSN: 01230670; e-ISSN: 2346-0911. Recuperado de:

http://revistas.uan.edu.co/index.php/papeles/article/view/225

Stenhouse, L. (2007). La investigación como base de la enseñanza. ISBN: 978-84-7112-315-2. Madrid, España: Ediciones Morata, S.L.

UNESCO (2015). Informe de la UNESCO sobre la ciencia, hacia 2030: resumen ejecutivo. ISBN: 978-3-940785-79-4. París, Francia: Organización de las Naciones Unidas para la Educación, la Ciencia y la Cultura. Recuperado de:

https://unesdoc.unesco.org/ark:/48223/pf0000235407 spa UNESCO (1998). Declaración Mundial sobre la Educación Superior en el siglo XXI: Visión y Acción. Código del documento: ED.98/CONF.202/3. París, Francia: Organización de la Naciones 
Unidas para la Educación, la Ciencia y la Cultura. Recuperado de: https://unesdoc.unesco.org/ark:/48223/pf0000113878 spa UPEL (2016). Manual de trabajos de Grado de Especialización y Maestría y Tesis Doctorales. 5ta. Edición, ISBN: 980-273-441-1. Caracas, Venezuela: Fondo Editorial de la Universidad Pedagógica Experimental Libertador - FEDUPEL.

Valencia, G. (2017). Plan de Capacitación en el Procesamiento Artesanal del Cambur como Actividad Productiva. Revista Scientific, 2(4), 157173, e-ISSN: 2542-2987. Recuperado de:

https://doi.org/10.29394/scientific.issn.2542-2987.2017.2.4.9.157-173 
OAI-PMH: http://www.indteca.com/ojs/index.php/Revista_Scientific/oai

Artículo Original / Original Article

Alirio Alejandro Tua García e-mail: aliriotua@gmail.com

Nacido en Barquisimeto, Venezuela, el 11 de enero del

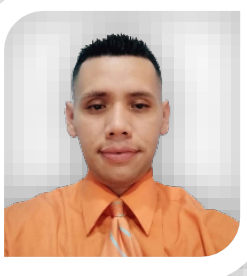
año 1989. Profesor en Electricidad Industrial; Magister en Investigación Educacional; Magister en Orientación de la Conducta; y Experto en Educación Virtual por la Fundación para la actualización tecnológica de Latinoamérica (FATLA); Docente de Metodología en el Centro de Investigaciones Psiquiátricas, Psicológicas y Sexológicas de Venezuela (CIPPSV), en Barquisimeto, estado Lara; Docente de la Unidad Educativa Nacional San Francisco Javier (UENSFJ); adscrito al Ministerio del Poder Popular para la Educación en Venezuela; Autor de diversos artículos publicados en revistas arbitradas.

El contenido de este manuscrito se difunde bajo una Licencia de Creative Commons ReconocimientoNoComercial-Compartirlgual 4.0 Internacional 